\title{
Two-Stage Time-Optimal Formation Reconfiguration Strategy *
}

\author{
Amir Ajorlou $^{\text {a }}$, Kaveh Moezzi ${ }^{\text {a }}$, Amir G. Aghdam ${ }^{\text {a }}$, Sergey G. Nersesov ${ }^{\text {b }}$ \\ ${ }^{a}$ Department of Electrical and Computer Engineering, Concordia University, 1455 De Maisonneuve Blvd. West \\ Montréal, Québec, H3G 1M8, Canada \\ ${ }^{\mathrm{b}}$ Department of Mechanical Engineering, Villanova University, 800 Lancaster Ave. Villanova, PA 19085, USA
}

\begin{abstract}
A time-optimal reconfiguration strategy for formation flying of autonomous acceleration-controlled agents is presented. In the proposed strategy, the agents are moved to a special designated formation in the time interval between the completion of the mission in the current formation and the issuance of the next reconfiguration command. It is shown that the problem of finding the special designated formation which minimizes the expected value of the reconfiguration time is nonconvex. This optimization problem is treated for two cases of constrained acceleration, and constrained acceleration and velocity. It is shown that in both cases, the search space for finding the special designated formation can be reduced to a convex compact set. An alternative search algorithm is presented for the second case, which consists of searching a vicinity of possible formations, and solving a convex nondifferentiable optimization problem. This search algorithm is typically much faster than the one concerning the acceleration constraint only. The effectiveness of the proposed strategy is illustrated via simulation.
\end{abstract}

Key words: Multi-agent systems; formation; optimization.

\section{Introduction}

The problem of formation flying control of autonomous agents has been extensively studied for various applications in the recent literature $[16,8,17,19,20,12,15]$. Such applications include, for instance, deep space missions, autonomous highway systems, and border patrol using unmanned aerial vehicles (UAV). The relative position of agents in a formation flying system may need to be reconfigured as changes occur in the mission. Such reconfiguration is desired to be carried out in such a way that some performance index such as maneuver time, fuel or energy consumption, etc. is minimized during reconfiguration. The choice of the performance index depends on the nature of the mission. For instance, while fuel consumption is the main concern in a deep-space mission to prolong the operation time [16], in some other applications such as rescue missions or forest fire surveillance [8] completing the mission in the shortest possible time is of utmost importance.

\footnotetext{
* This work has been supported in part by the Natural Sciences and Engineering Research Council of Canada (NSERC) under Grant STPGP-364892-08.

Email addresses: a jorlou@gmail.com (Amir Ajorlou), kavehmoezi@gmail.com (Kaveh Moezzi), aghdameece.concordia.ca (Amir G. Aghdam), sergey . nersesov@villanova. edu (Sergey G. Nersesov).
}

Fuel, energy and time are among the most important quantities of interest in a multi-agent system, and a number of reconfiguration strategies are reported in the literature to minimize them. $[3,18,13]$ propose minimum-fuel control strategies for spacecraft and micro-satellites under various constraints such as balanced fuel consumption and fixed mission completion time. Minimizing energy for network reconfiguration is investigated in [11,10,22], where reconfiguration strategies are proposed for multiple aircraft and deep-space spacecraft. $[23,1]$ introduce a strategy to properly coordinate the agents in the time-interval between the accomplishment of the mission at the most recent formation and the issuance of the next reconfiguration command, which will henceforth be referred to as the idle time, in order to achieve better reconfiguration performance. This is carried out by moving the agents to a special designated formation during the idle time. This special designated formation in [23] has the property that it positions each agent as close as possible to its next potential location, aiming to minimize the average reconfiguration time. The special designated formation in [1], on the other hand, is obtained in such a way that the expected value of the reconfiguration energy consumption for a team of acceleration-controlled agents is minimized.

In this paper, a two-stage time-optimal reconfiguration strategy for formation flying of a team of autonomous acceleration-controlled agents is presented. The results de- 
veloped here are the time-optimal counterparts of the ones in [1], where a two-stage energy-optimal reconfiguration strategy is presented for a multi-agent system. A special designated formation is obtained analogously to [1], such that the expected value of the time required to move the team from this formation to a newly commanded formation is minimized. The proposed strategy can be effectively used in applications where the reconfiguration time is a critical component of the formation mission. Such applications include border patrol, or defending a base station by forming different configurations depending on the potential enemy threat. Unlike the energy-optimal case presented in [1], it turns out that the problem of finding the time-optimal special designated formation is a nonconvex nondifferentiable optimization problem. This problem is treated for two cases of constrained acceleration, and constrained acceleration and velocity. It is shown that in both cases the search space for finding the special designated formation can be reduced to a convex compact set. In the case when both acceleration and velocity are constrained, an alternative algorithm is presented which involves searching a vicinity of possible formations, and solving a convex nondifferentiable optimization problem. This simplified search method is typically much faster than the original one. Sufficient conditions are provided under which the special designated formation can be directly obtained from the solution of the convex optimization problem. A norm-bounded subgradient is derived for the convex nondifferentiable objective function in the case of constrained acceleration and velocity, which is subsequently used to solve the problem numerically.

The rest of the paper is structured as follows. In Section 2 , some notations and definitions are introduced which will prove convenient in presenting the main results. The twostage time-optimal formation reconfiguration strategy is provided in Section 3. Simulation results are presented in Section 4, which demonstrate the effectiveness of the proposed approach. Section 5 outlines the conclusions, and finally the proofs of the lemmas are provided in the appendix (Section 6).

\section{Notations and Definitions}

Definition 1 Consider a multi-agent system whose configuration can vary from time to time. The time between the completion of the mission at the current formation and the issuance of the next reconfiguration command is referred to as the idle time, and is denoted by $\tau$. Furthermore, the time it takes for the agents to move from the current formation to a newly commanded formation is called the reconfiguration time, and is denoted by $T$.

Let $\mathscr{D}$ denote the set of predefined desired formation configurations, which is assumed to be finite. Any $d \in \mathscr{D}$ is described as $d=\left[d_{1}^{T}, d_{2}^{T}, \ldots, d_{n}^{T}\right]^{T}, d \in \mathbb{R}^{3 n}$, where $n$ is the number of agents and $d_{j} \in \mathbb{R}^{3}$ is the position of agent $j$ in the formation $d$ with respect to an inertial reference frame.
The union of all possible positions for agent $j$ in $\mathscr{D}$ constitutes a region $\mathscr{D}_{j}$ for this agent, i.e. $\mathscr{D}_{j}=\cup_{d \in \mathscr{D}} d_{j}$. In general, some formations may occur more frequently than the others. To formulate this, the probability of the formation $d$ is denoted by $p(d), d \in \mathscr{D}$, which is assumed to be known $a$ priori. Note that by definition $p(d) \geq 0$ and $\sum_{d \in \mathscr{D}} p(d)=1$. Let the set of integers $\{1,2, \ldots, n\}$ be denoted by $\mathbb{N}_{n}$. The agents are assumed to have double integrator dynamics of the form $\ddot{x}_{j}=a_{j}, j \in \mathbb{N}_{n}$, i.e., each agent is controlled by its acceleration (or more precisely by applying a force). The initial and final velocities of the agents while moving from their current formation to a newly commanded formation are assumed to be zero with respect to the reference frame.

It is desired in the next section to find a control strategy under which the expected value of the reconfiguration time is minimized.

\section{Two-Stage Time-Optimal Formation Reconfigura- tion Strategy}

The objective of the two-stage time-optimal formation reconfiguration strategy is to move the agents to a special formation during the idle time, in such a way that the expected value of the reconfiguration time, i.e. the time required to move the team from this formation to a newly commanded formation is minimized. This special formation will be referred to as the time-optimal special designated formation (TOSDF) and will be denoted by $\mathscr{S}_{T} \in \mathbb{R}^{3 n}$. It is assumed that the idle time is long enough for the agents to move from any possible formation to their location in $\mathscr{S}_{T}$. The cost function to be minimized is

$$
J(x ; T)=\sum_{d \in \mathscr{D}} p(d) \max _{1 \leq j \leq n} T\left(x_{j}, d_{j}\right)
$$

where $T\left(x_{j}, d_{j}\right)$ is the minimum time required for agent $j$ to move from $x_{j}$ to $d_{j}$ with zero initial and final velocities, and $x=\left[x_{1}^{T}, x_{2}^{T}, \ldots, x_{n}^{T}\right]^{T}$.

Let $a_{j}(\cdot) \in \mathbb{R}^{3}$ and $v_{j}(\cdot) \in \mathbb{R}^{3}$ be the acceleration and velocity of agent $j$ while moving from $x_{j}$ to $d_{j}$. The underlying optimization problem is investigated for two cases. In the first case, a threshold on the acceleration of every agent is considered as $\left\|a_{j}\right\| \leq a_{\max }, j \in \mathbb{N}_{n}$. In the second case, thresholds for both acceleration and velocity are considered, i.e. $\left\|a_{j}\right\| \leq a_{\max }$ and $\left\|v_{j}\right\| \leq v_{\max }$, for all $j \in \mathbb{N}_{n}$. It will be shown later that both cases share a number of interesting properties. A few lemmas are presented in the sequel, which will be used to derive the results for both cases. The proofs of these lemmas are provided in the appendix.

Remark 1 It is to be noted that in the conventional onestage strategy, the agents stay at their most recent formation during the idle time. The initial configuration of the agents is random (with exactly the same set of possible formations $\mathscr{D}$ and probabilities $p(d), d \in \mathscr{D})$. This is the basis for the 
comparison of the reconfiguration time in the proposed twostage strategy and the conventional one-stage strategy.

Remark 2 Finding the TOSDF requires a closed-form analytical expression for the reconfiguration time, i.e. the time required to move the agents from formation $x \in \mathbb{R}^{3 n}$ to $d \in \mathscr{D}$. With the collision avoidance constraint, however, a closedform expression for this function may not exist. Since the set of potential formations is assumed to be known and the TOSDF is computed off-line, therefore any pair of crossing optimal trajectories obtained for the resultant TOSDF (i.e. the trajectories which meet at the same point in time) can be slightly modified to avoid collision (with no major impact on the reconfiguration time).

Note 1 In order to reduce the amount of backtracking, all notations used in developing the time-optimal formation reconfiguration strategy are listed in Table 1.

Lemma 1 Consider the function $f: \mathbb{R}^{3 n} \rightarrow \mathbb{R}$ defined as $f(x)=\sum_{d \in \mathscr{D}} p(d) \max _{1 \leq j \leq n} g\left(x_{j}-d_{j}\right)$, where $g: \mathbb{R}^{3} \rightarrow \mathbb{R}$ satisfies the following conditions

i) $g(0)=0$ and $g(r)>0$ for any nonzero $r \in \mathbb{R}^{3}$.

ii) $g^{\prime}(0 ; v)=+\infty$ for any nonzero direction $v \in \mathbb{R}^{3}$.

iii) $\left\|g^{\prime}(r ; v)\right\|<\infty$ for any nonzero $r$.

Then, every $x \in \mathscr{D}$ is a local minimizer of $f$.

Lemma 2 Consider the function $f$ defined in Lemma 1, and assume that $g$ satisfies the following conditions

i) $g(0)=0$ and $g(r) \geq 0$

ii) $g(r)=g(-r)$

iii) $g(r)+g(s) \geq g(r+s)$

Suppose that $p(\tilde{d}) \geq 0.5$ for some $\tilde{d} \in \mathscr{D}$. Then, $x=\tilde{d}$ is a global minimizer of the function $f$.

Lemma 3 Let $\mathscr{C}_{j} \subset \mathbb{R}^{3}$ denote the convex hull of the region $\mathscr{D}_{j}$ corresponding to agent $j$, for any $j \in \mathbb{N}_{n}$. Consider the function $f$ defined in Lemma 1 , and assume that $g(r)$ is an increasing function of $\|r\|$, where $\|\cdot\|$ is the Euclidean norm. Then, $f$ has a global minimizer lying in $\mathscr{C}=\mathscr{C}_{1} \times \mathscr{C}_{2} \times \ldots \times$ $\mathscr{C}_{n}$.

Lemma 4 Let $x^{*}$ be a global minimizer of the function $f$ defined in Lemma 1, and suppose that $g$ satisfies the conditions of Lemma 2. Then,

$$
0.5 \sum_{d \in \mathscr{D}} p(d) f(d) \leq f\left(x^{*}\right) \leq \sum_{d \in \mathscr{D}} p(d) f(d)
$$

\subsection{Bounded acceleration}

It is assumed now that the magnitude of the acceleration of each agent is bounded by $a_{\max }$. Let $T_{1}\left(x_{j}, d_{j}\right)$ denote
Table 1

List of the notations used in developing the two-stage time-optimal formation reconfiguration strategy.

\begin{tabular}{|c|c|}
\hline Notation & Description \\
\hline $\mathscr{S}_{T}$ & time-optimal special designated formation \\
\hline $\mathscr{D}$ & set of predefined desired formation configurations \\
\hline $\mathscr{D}_{j}$ & union of all possible positions for agent $j$ in $\mathscr{D}$ \\
\hline $\mathscr{C}_{j}$ & convex hull of the region $\mathscr{D}_{j}$ \\
\hline $\mathscr{C}$ & $\mathscr{C}_{1} \times \mathscr{C}_{2} \times \ldots \times \mathscr{C}_{n}$ \\
\hline$p(d)$ & probability of $d$ being the desired formation \\
\hline$d_{j}$ & position of agent $j$ in formation $d$ \\
\hline$a_{j}(t)$ & acceleration of agent $j$ moving from $x_{j}$ to $d_{j}$ \\
\hline$v_{j}(t)$ & velocity of agent $j$ moving from $x_{j}$ to $d_{j}$ \\
\hline$a_{\max }$ & threshold on the acceleration of every agent \\
\hline$v_{\max }$ & threshold on the velocity of every agent \\
\hline$L$ & $\frac{v_{\max }^{2}}{a_{\max }}$ \\
\hline $\mathscr{B}_{d}^{\delta}$ & $\delta$-vicinity of formation $d$ \\
\hline $\mathscr{D}^{L}$ & union of all $\mathscr{B}_{d}^{L}$, s for $d \in \mathscr{D}$ \\
\hline$T\left(x_{j}, d_{j}\right)$ & $\begin{array}{l}\text { minimum time required for agent } j \text { to move from } \\
x_{j} \text { to } d_{j} \text { with zero initial and final velocities }\end{array}$ \\
\hline$T_{1}\left(x_{j}, d_{j}\right)$ & $\begin{array}{l}\text { minimum time required for agent } j \text { to move from } \\
x_{j} \text { to } d_{j} \text { with zero initial and final velocities, sub- } \\
\text { ject to bounded acceleration }\end{array}$ \\
\hline$T_{2}\left(x_{j}, d_{j}\right)$ & $\begin{array}{l}\text { minimum time required for agent } j \text { to move from } \\
x_{j} \text { to } d_{j} \text { with zero initial and final velocities, sub- } \\
\text { ject to bounded acceleration and bounded velocity }\end{array}$ \\
\hline$T_{3}\left(x_{j}, d_{j}\right)$ & $\begin{array}{l}\text { minimum time required for agent } j \text { to move from } \\
x_{j} \text { to } d_{j} \text { with zero initial and final velocities, sub- } \\
\text { ject to bounded acceleration and bounded velocity } \\
\text { for the case when }\left\|x_{j}-d_{j}\right\|>L\end{array}$ \\
\hline$T^{(a)}$ & $\begin{array}{l}\text { expected value of the reconfiguration time for the } \\
\text { two-stage time-optimal strategy with bounded ac- } \\
\text { celeration }\end{array}$ \\
\hline$T^{(v a)}$ & $\begin{array}{l}\text { expected value of the reconfiguration time for the } \\
\text { two-stage time-optimal strategy with bounded ac- } \\
\text { celeration and bounded velocity }\end{array}$ \\
\hline$T^{(1 a)}$ & $\begin{array}{l}\text { expected value of the reconfiguration time for the } \\
\text { conventional one-stage strategy with bounded ac- } \\
\text { celeration }\end{array}$ \\
\hline$T^{(1 v a)}$ & $\begin{array}{l}\text { expected value of the reconfiguration time for the } \\
\text { conventional one-stage strategy with bounded ac- } \\
\text { celeration and bounded velocity }\end{array}$ \\
\hline
\end{tabular}

the minimum time required by agent $j$ to move from $x_{j}$ to $d_{j}$ with zero initial and final velocities under the above constraint. Using Pontryagin's minimum principle, it can be easily verified that a time-optimal strategy will require $a_{j}(t)=\operatorname{sgn}\left(\frac{T_{1}}{2}-t\right) a_{\max } \frac{d_{j}-x_{j}}{\left\|x_{j}-d_{j}\right\|}$. Substituting for $a_{j}(\cdot)$ in 
$\int_{0}^{T_{1}} \int_{0}^{\tau} a_{j}(t) d t d \tau=d_{j}-x_{j}$ and solving for $T_{1}$, one will obtain

$$
T_{1}\left(x_{j}, d_{j}\right)=\frac{2}{\sqrt{a_{\max }}} \sqrt{\left\|x_{j}-d_{j}\right\|}
$$

Thus, the function to be minimized is

$$
J\left(x ; T_{1}\right)=\frac{2}{\sqrt{a_{\max }}} \sum_{d \in \mathscr{D}} p(d) \max _{j \in \mathbb{N}_{n}} \sqrt{\left\|x_{j}-d_{j}\right\|}
$$

which shows that finding the TOSDF involves a nonconvex nondifferentiable optimization problem. However, one can use Lemmas 1-4 to characterize some interesting properties of this optimization problem. These properties are presented in the following theorem.

Theorem 1 Consider the function $J\left(x ; T_{1}\right)$, where $T_{1}\left(x_{j}, d_{j}\right)$ is given by (3). The following statements are all true.

i) Every $d \in \mathscr{D}$ is a local minimizer of $J$.

ii) If $p(\tilde{d}) \geq 0.5$ for some $\tilde{d} \in \mathscr{D}$, then $\tilde{d}$ is a global minimizer of $J$.

iii) $J$ has a global minimizer lying in $\mathscr{C}=\mathscr{C}_{1} \times \mathscr{C}_{2} \times \ldots \times$ $\mathscr{C}_{n}$, where $\mathscr{C}_{j}$ denotes the convex hull of the region $\mathscr{D}_{j}$ for agent $j, j \in \mathbb{N}_{n}$.

Proof.

Part (i). Let $g(r)=\sqrt{\|r\|}, r \in \mathbb{R}^{3}$. Then, for any direction $v$ and nonzero $r$

$$
g^{\prime}(r ; v)=\frac{v^{T} r}{2\|r\|^{\frac{3}{2}}}
$$

It is now easy to show that $g$ satisfies the conditions of Lemma 1, which completes the proof of part (i).

Part (ii). The proof follows immediately from Lemma 2.

Part (iii). The proof is straightforward using Lemma 3.

One of the results of Theorem 1 is that $J$ has at least $|\mathscr{D}|$ local minimizers. Because of the multiple local minima of the function $J$ (given by (4)), methods of descent for nondifferentiable optimization (e.g., [14,2]) may converge to a suboptimal solution. However, part (iii) of the theorem reduces the search space for finding the TOSDF to the convex compact set $\mathscr{C}=\mathscr{C}_{1} \times \mathscr{C}_{2} \times \ldots \times \mathscr{C}_{n}$. It is to be noted that this search can be tedious in general and may take a relatively long time to complete. However, this is not a major concern since the TOSDF is computed off-line.

In the next theorem, an upper bound is presented on the maximum reduction in the reconfiguration time using the proposed two-stage strategy.

Theorem 2 Let $T^{(a)}$ and $T^{(1 a)}$ denote the expected values of the reconfiguration time for the two-stage time-optimal strategy with the maximum acceleration constraint $\left\|a_{j}\right\| \leq a_{\max }$, $j \in \mathbb{N}_{n}$, and the conventional one-stage counterpart with the same constraint, respectively. Then $0.5 T^{(1 a)} \leq T^{(a)} \leq T^{(1 a)}$.
Proof. Let $g(r)=\sqrt{\|r\|}$ for any $r \in \mathbb{R}^{3}$. Let also $f$ be defined as in Lemma 1. Then, the function $J$ given by (4) can be written as $J\left(x ; T_{1}\right)=\frac{2}{\sqrt{a_{\max }}} f(x)$. Since $T^{(a)}$ is the minimum of $J\left(x ; T_{1}\right)$, hence $T^{(a)}=\frac{2}{\sqrt{a_{\max }}} f\left(x^{*}\right)$, where $x^{*}$ is a global minimizer of $f$. In the conventional one-stage strategy, the agents stay at their most recent formation during the idle time. Therefore, the expected value of the reconfiguration time given the current formation $d$ is $J\left(d ; T_{1}\right)$, and hence $T^{(1 a)}=\sum_{d \in \mathscr{D}} p(d) J\left(d ; T_{1}\right)=\frac{2}{\sqrt{a_{\max }}} \sum_{d \in \mathscr{D}} p(d) f(d)$. On the other hand, it can be easily verified that $g$ satisfies the conditions given in Lemma 2. Thus, from Lemma 4, $0.5 \sum_{d \in \mathscr{D}} p(d) f(d) \leq f\left(x^{*}\right) \leq \sum_{d \in \mathscr{D}} p(d) f(d)$, which completes the proof.

\subsection{Bounded acceleration and bounded velocity}

Assume now that in addition to the constraint on the acceleration, the maximum velocity of each agent is also bounded by $v_{\text {max }}$. Let $T_{2}\left(x_{j}, d_{j}\right)$ denote the minimum time required by agent $j$ to move from $x_{j}$ to $d_{j}$ with zero initial and final velocities under these constraints. One can use Pontryagin's minimum principle to find $T_{2}$ in this case. To this end, define

$$
L=\frac{v_{\max }^{2}}{a_{\max }}
$$

If $\left\|x_{j}-d_{j}\right\| \leq L$, then a time-optimal strategy will require

$$
a_{j}(t)=\operatorname{sgn}\left(\frac{T_{2}}{2}-t\right) a_{\max } \frac{d_{j}-x_{j}}{\left\|x_{j}-d_{j}\right\|}
$$

If on the other hand $\left\|x_{j}-d_{j}\right\|>L$, then the constraint on the velocities affects the equations, and the time-optimal control will be

$$
a_{j}(t)= \begin{cases}a_{\max } \frac{d_{j}-x_{j}}{\left\|x_{j}-d_{j}\right\|}, & 0 \leq t \leq \frac{v_{\max }}{a_{\max }} \\ 0, & \frac{v_{\max }}{a_{\max }}<t \leq T_{2}-\frac{v_{\max }}{a_{\max }} \\ -a_{\max } \frac{d_{j}-x_{j}}{\left\|x_{j}-d_{j}\right\|}, & T_{2}-\frac{v_{\max }}{a_{\max }}<t \leq T_{2}\end{cases}
$$

Substituting for $a_{j}(\cdot)$ from (7) and (8) in the relation $\int_{0}^{T_{2}} \int_{0}^{\tau} a_{j}(t) d t d \tau=d_{j}-x_{j}$ leads to an equation in terms of $T_{2}$ whose solution is

$$
T_{2}\left(x_{j}, d_{j}\right)= \begin{cases}\frac{2}{\sqrt{a_{\max }}} \sqrt{\left\|x_{j}-d_{j}\right\|}, & \left\|x_{j}-d_{j}\right\| \leq L \\ \frac{\left\|x_{j}-d_{j}\right\|}{v_{\max }}+\frac{v_{\max }}{a_{\max }}, & \left\|x_{j}-d_{j}\right\|>L\end{cases}
$$

The function to be minimized is (see (1))

$$
J\left(x ; T_{2}\right)=\sum_{d \in \mathscr{D}} p(d) \max _{j \in \mathbb{N}_{n}} T_{2}\left(x_{j}, d_{j}\right)
$$


Minimizing $J\left(x ; T_{2}\right)$ (with $T_{2}\left(x_{j}, d_{j}\right)$ given by (9)) is still a nonconvex nondifferentiable optimization problem. However, it is shown in the sequel that the results presented for the case of bounded acceleration are also valid in this case.

Theorem 3 Consider the function $J\left(x ; T_{2}\right)$, where $T_{2}\left(x_{j}, d_{j}\right)$ is given by (9). The following statements are true.

i) Every $d \in \mathscr{D}$ is a local minimizer of $J$.

ii) Suppose that $p(\tilde{d}) \geq 0.5$, for some $\tilde{d} \in \mathscr{D}$. Then, $\tilde{d}$ is a global minimizer of $J$.

iii) $J$ has a global minimizer lying in $\mathscr{C}=\mathscr{C}_{1} \times \mathscr{C}_{2} \times \ldots \times$ $\mathscr{C}_{n}$, where $\mathscr{C}_{j}$ denotes the convex hull of the region $\mathscr{D}_{j}$ for agent $j, j \in \mathbb{N}_{n}$.

Proof.

Part (i). For any $r \in \mathbb{R}^{3}$, let

$$
g(r)= \begin{cases}\frac{2}{\sqrt{a_{\max }}} \sqrt{\|r\|}, & \|r\| \leq L \\ \frac{\|r\|}{v_{\max }}+\frac{v_{\max }}{a_{\max }}, & \|r\|>L\end{cases}
$$

For any direction $v$ and nonzero vector $r$

$$
g^{\prime}(r ; v)= \begin{cases}\frac{v^{T} r}{\sqrt{a_{\max }}\|r\|^{\frac{3}{2}}}, & \|r\| \leq L \\ \frac{v^{T} r}{v_{\max }\|r\|}, & \|r\|>L\end{cases}
$$

It is to be noted that for $\|r\|=L, \frac{v^{T} r}{\sqrt{a_{\max }} L^{\frac{3}{2}}}=\frac{v^{T} r}{v_{\max } L}$ since $L=\frac{v_{\max }^{2}}{a_{\max }}$. It is easy to show that $g$ satisfies the conditions of Lemma 1, and the proof follows directly from that.

Part (ii). To prove this part, it suffices to show that the function $g$ in (11) satisfies the conditions of Lemma 2. It is straightforward to show that the first two conditions hold. To prove that the third condition also holds, some useful properties of $g$ are presented first. It can be easily verified that $\frac{\|r\|}{v_{\max }} \leq g(r) \leq \frac{\|r\|}{v_{\max }}+\frac{v_{\max }}{a_{\max }}$. Moreover, if $\|r\| \leq L$, then $g(r) \geq 2 \frac{\|r\|}{v_{\max }}$, and if $\|r\|>L$, then $g(r)<2 \frac{\|r\|}{v_{\max }}$. Condition (iii) of Lemma 2 will now be proved by considering two cases:

Case (1): $\|r\| \leq L$ AND $\|s\| \leq L$. If $\|r+s\| \leq L$, then clearly $g(r)+g(s) \geq g(r+s)$. If $\|r+s\|>L$, then

$$
\begin{aligned}
g(r)+g(s) & \geq 2 \frac{\|r\|}{v_{\max }}+2 \frac{\|s\|}{v_{\max }} \\
& \geq 2 \frac{\|r+s\|}{v_{\max }} \\
& >g(r+s)
\end{aligned}
$$

Case (2): $\|r\|>L$ OR $\|s\|>L$. Assume $\|r\|>L$; then

$$
\begin{aligned}
g(r)+g(s) & \geq \frac{\|r\|}{v_{\max }}+\frac{v_{\max }}{a_{\max }}+\frac{\|s\|}{v_{\max }} \\
& \geq \frac{\|r+s\|}{v_{\max }}+\frac{v_{\max }}{a_{\max }} \\
& \geq g(r+s)
\end{aligned}
$$

Part (iii). The proof follows directly from Lemma 3.

Part (iii) of the above theorem suggests that one can find a global minimizer of $J\left(x ; T_{2}\right)$ by searching only the convex compact set $\mathscr{C}=\mathscr{C}_{1} \times \mathscr{C}_{2} \times \ldots \times \mathscr{C}_{n}$. As also mentioned earlier, such a search can be tedious in general. A more time-efficient search algorithm is developed in the sequel. For any formation $d \in \mathscr{D}$ and positive real number $\delta$, define the $\delta$-vicinity of $d$ as

$$
\mathscr{B}_{d}^{\delta}=\left\{x \in \mathbb{R}^{3 n} \mid \max _{1 \leq j \leq n}\left\|x_{j}-d_{j}\right\| \leq \delta\right\}
$$

This is, in fact, the set of all formations in which the position of agent $j, j \in \mathbb{N}_{n}$, is selected from a ball of radius $\delta$, centered at the position of this agent in the formation $d$. The following theorem presents an alternative search algorithm which involves searching the $L$-vicinity of the formations in $\mathscr{D}$. It is to be noted that this is typically a much smaller set compared to $\mathscr{C}$. The algorithm also involves a convex optimization problem which can be solved efficiently using existing methods.

Theorem 4 Let $\mathscr{D}^{L}=\cup_{d \in \mathscr{D}} \mathscr{B}_{d}^{L}$, where $\mathscr{B}_{d}^{L}$ is the L-vicinity of the formation $d$, and $L$ is given by (6). Suppose that $x^{(2)}$ is a minimizer of $J\left(x ; T_{2}\right)$ over $\mathscr{D}^{L} \cap \mathscr{C}$, and that $x^{(3)}$ is a global minimizer of $J\left(x ; T_{3}\right)$, where

$$
T_{3}\left(x_{j}, d_{j}\right)=\frac{\left\|x_{j}-d_{j}\right\|}{v_{\max }}+\frac{v_{\max }}{a_{\max }}
$$

If $J\left(x^{(2)} ; T_{2}\right) \leq J\left(x^{(3)} ; T_{3}\right)$, then $x^{(2)}$ is a global minimizer of $J\left(x ; T_{2}\right)$; otherwise, $x^{(3)}$ is a global minimizer of $J\left(x ; T_{2}\right)$.

Proof. Following an approach similar to the one used in the proof of Lemma 3, one can show that $x^{(2)}$ is a minimizer of $J\left(x ; T_{2}\right)$ on $\mathscr{D}^{L}$. If $x^{(3)} \notin \mathscr{D}^{L}$, then $x^{(3)}$ is a minimizer of $J\left(x ; T_{2}\right)$ on $\mathbb{R}^{3 n} \backslash \mathscr{D}^{L}$ because $J\left(x ; T_{2}\right)=J\left(x ; T_{3}\right)$ for $x \in$ $\mathbb{R}^{3 n} \backslash \mathscr{D}^{L}$, and the proof follows directly from that. If on the other hand $x^{(3)} \in \mathscr{D}^{L}$, then for any $x \in \mathbb{R}^{3 n} \backslash \mathscr{D}^{L}$

$$
\begin{aligned}
J\left(x ; T_{3}\right) & \geq J\left(x^{(3)} ; T_{3}\right) \\
& \geq J\left(x^{(3)} ; T_{2}\right) \\
& \geq J\left(x^{(2)} ; T_{2}\right)
\end{aligned}
$$

(note that the relation $T_{3}\left(x_{j}, d_{j}\right) \geq T_{2}\left(x_{j}, d_{j}\right)$ is used to derive (17)). Therefore, in this case $x^{(2)}$ is a global minimizer of $J\left(x ; T_{2}\right)$. 
Finding a global minimizer of $J\left(x ; T_{3}\right)$ for $T_{3}\left(x_{j}, d_{j}\right)$ given in (16) is a convex optimization problem, and $\mathscr{D}^{L} \cap \mathscr{C}$ is typically much smaller than $\mathscr{C}$ (specially when $L$ is small compared to the diameter of the region $\mathscr{C}_{j}, j \in \mathbb{N}_{n}$ ). Consequently, a search algorithm based on Theorem 4 could be much more efficient than any algorithm that searches over the whole space $\mathscr{C}$. There are certain cases as described in the next theorem, where it may not even be required to search $\mathscr{D}^{L} \cap \mathscr{C}$.

Theorem 5 Suppose that $x^{\mathscr{D}}$ is a minimizer of $J\left(x ; T_{2}\right)$ on $x \in \mathscr{D}$. If $x^{(3)}$ is a global minimizer of $J\left(x ; T_{3}\right)$ and

$$
J\left(x^{(3)} ; T_{3}\right) \leq J\left(x^{\mathscr{D}} ; T_{2}\right)-2 \frac{v_{\max }}{a_{\max }}
$$

then $x^{(3)}$ is a global minimizer of $J\left(x ; T_{2}\right)$ as well.

Proof. Let $x^{(2)}$ be a minimizer of $J\left(x ; T_{2}\right)$ on $\mathscr{D}^{L}$ as defined in Theorem 4. Then, $x^{(2)} \in \mathscr{B}_{d}^{L}$ for some $d \in \mathscr{D}$. Let also $G(x)=\max _{1 \leq j \leq n} g\left(x_{j}\right)$, for the $g$ given in (11). Following an approach similar to the one used in the proof of Lemma 4, one can show that

$$
J\left(d ; T_{2}\right) \leq J\left(x^{(2)} ; T_{2}\right)+G\left(d-x^{(2)}\right)
$$

On the other hand, $x^{(2)} \in \mathscr{B}_{d}^{L}$ yields $G\left(d-x^{(2)}\right) \leq 2 \sqrt{\frac{L}{a_{\max }}}=$ $2 \frac{v_{\max }}{a_{\max }}$. This relation along with (18) and (19) implies that $J\left(x^{(3)} ; T_{3}\right) \leq J\left(x^{(2)} ; T_{2}\right)$, and the proof is completed using Theorem 4 .

Remark 3 It follows from the above proof that a quick alternative to the exact solution of the TOSDF problem in case of constrained acceleration and velocity could be either $x^{D}$ or $x^{(3)}$, whichever leads to a smaller performance index. This means that without searching the L-vicinity of the formations, one can obtain a two-stage strategy whose corresponding reconfiguration time exceeds the minimum value by at most $2 \frac{v_{\max }}{a_{\max }}$.

Minimizing $J\left(x ; T_{3}\right)$ involves a convex nondifferentiable optimization problem. The subgradient method $[21,5,4]$ can be employed now to numerically solve this optimization problem. In this method, it is required to compute a normbounded subgradient for the objective function at any point in its domain. Basic rules for finding a subgradient are discussed in $[5,4,7]$, and are summarized in the next remark.

Remark 4 Consider a convex function $\phi: \mathbb{R}^{n} \rightarrow \mathbb{R}$.

(1) If $\phi$ is differentiable at $x$, then its gradient at $x$ is the only element of the subdifferential $\partial \phi(x)$.

(2) For any scalar $\omega \geq 0$, a subgradient of $\omega \phi$ at $x$ is given by $\omega \xi$, where $\xi$ is any subgradient of $\phi$ at $x$.

(3) If $\phi(x)=\phi_{1}(x)+\ldots+\phi_{m}(x)$, where $\phi_{1}, \ldots, \phi_{m}$ are convex, then any $\xi$ of the form $\xi=\xi_{1}+\ldots+\xi_{m}$ is in $\partial \phi(x)$, where $\xi_{i} \in \partial \phi_{i}(x)$ for all $i \in \mathbb{N}_{m}$.
(4) Suppose that $\phi(x)=\max _{1 \leq i \leq m} \phi_{i}(x)$, where $\phi_{1}, \ldots, \phi_{m}$ are convex. Then,

$$
\partial \phi(x)=\operatorname{Conv}\left(\cup\left\{\partial \phi_{i}(x) \mid \phi_{i}(x)=\phi(x)\right\}\right)
$$

where $\operatorname{Conv}(\cdot)$ denotes the convex hull of $(\cdot)$.

The next theorem presents a norm-bounded subgradient of $J\left(x ; T_{3}\right)$ at any $x \in \mathbb{R}^{3 n}$ using Remark 4.

Theorem 6 Consider the function $J\left(x ; T_{3}\right)$, where $T_{3}$ is defined in (16). For any $x \in \mathbb{R}^{3 n}$ and any agent $j \in \mathbb{N}_{n}$, define $\xi_{j} \in \mathbb{R}^{3}$ as

$$
\xi_{j}=\frac{1}{v_{\max }} \sum_{\substack{d \in \mathscr{O}-\{x\} \\ j \in I_{d}}} \frac{p(d)}{\left|I_{d}\right|} \cdot \frac{x_{j}-d_{j}}{\left\|x_{j}-d_{j}\right\|}
$$

where $I_{d}$ denotes the set of active indices at $x$ for formation $d$, defined as $I_{d}=\left\{\bar{j} \mid\left\|x_{\bar{j}}-d_{\bar{j}}\right\|=\max _{1 \leq j \leq n}\left\|x_{j}-d_{j}\right\|\right\}$. Let $\xi_{j}$ be zero if $j$ is not active at any formation, and define $\xi=\left[\xi_{1}^{T}, \ldots, \xi_{n}^{T}\right]^{T}$. Then, $\xi$ is a subgradient of $J\left(x ; T_{3}\right)$ and $\|\xi\| \leq \frac{1}{v_{\max }}$.

Proof. Define $G(x)=\max _{j \in \mathbb{N}_{n}} T_{3}\left(x_{j}, 0\right)$; then, $J\left(x ; T_{3}\right)=$ $\sum_{d \in \mathscr{D}} p(d) G(x-d)$. It is easy to check that 0 is a subgradient of $G(x-d)$ at $x=d$. Now, assume that $x \neq d$, and for any $j \in I_{d}$ denote with $\xi_{j_{d}}$ a subgradient of $T_{3}\left(x_{j}, d_{j}\right)$ at $x_{j}$. The inequality $x \neq d$ implies that $x_{j} \neq d_{j}$ for any $j \in I_{d}$. The function $T_{3}\left(x_{j}, d_{j}\right)$ is differentiable for $x_{j} \neq d_{j}$, and hence Remark 4 implies that $\xi_{j_{d}}=\frac{x_{j}-d_{j}}{v_{\max }\left\|x_{j}-d_{j}\right\|}$, which is the gradient of $T_{3}$ at $x_{j}$. Now, let $\xi_{j_{d}}$ be zero for any $j \notin I_{d}$. According to Remark 4, $\xi_{d}=\frac{1}{\left|I_{d}\right|}\left[\xi_{1_{d}}^{T}, \ldots, \xi_{n_{d}}^{T}\right]^{T}$ is a subgradient of $G(x-d)$ at $x$. The fact that $\xi_{j}=\sum_{d \in \mathscr{D}-\{x\}} p(d) \xi_{j_{d}}$ along with Remark 4 implies that $\xi$ defined above is a subgradient of $J\left(x ; T_{3}\right)$ at $x$. Finally,

$$
\begin{aligned}
\|\xi\| & \leq \sum_{j=1}^{n}\left\|\xi_{j}\right\| \\
& \leq \frac{1}{v_{\max }} \sum_{j=1}^{n} \sum_{\substack{d \in \mathscr{D}-\{x\} \\
j \in I_{d}}} \frac{p(d)}{\left|I_{d}\right|}=\frac{1}{v_{\max }} \sum_{d \in \mathscr{D}-\{x\}} p(d) \\
& \leq \frac{1}{v_{\max }}
\end{aligned}
$$

In the next theorem, an upper bound is presented (similarly to Theorem 2) on the maximum reduction in the reconfiguration time with the proposed two-stage strategy in the case of bounded acceleration and velocity.

Theorem 7 Let $T^{(v a)}$ and $T^{(1 v a)}$ denote the expected values of the reconfiguration time for the two-stage time-optimal strategy with bounded acceleration and velocity, and its 
conventional one-stage counterpart, respectively. Then $0.5 T^{(1 v a)} \leq T^{(v a)} \leq T^{(1 v a)}$.

Proof. The proof is similar to that of Theorem 2, and follows immediately on noting that the function $g$ defined in (11) satisfies the conditions of Lemma 2, as shown in Theorem 3.

\section{Simulation Results}

In this section, the effectiveness of the proposed two-stage time-optimal strategy is illustrated by simulation.

Consider a team of four acceleration-controlled agents, and let the set of possible formations $\mathscr{D}$ consist of six entries $d_{1}, \ldots, d_{6}$ given below, with the same probabilities $p\left(d_{i}\right)=\frac{1}{6}$ for $i=1, \ldots, 6$.

$$
\begin{aligned}
d_{1}= & {\left[\begin{array}{lllllll}
0.33 & 0 & 0.94, & -0.16 & 0.28 & 0.94, \\
& -0.16 & -0.28 & 0.94, & 0 & 0 & 1.41
\end{array}\right]^{T} } \\
d_{2}= & {\left[\begin{array}{lllllll}
0 & -0.57 & 0, & -0.5 & -0.28 & 0, \\
& -0.5 & -0.86 & 0, & -0.33 & -0.57 & 0.47
\end{array}\right]^{T} } \\
d_{3}= & {\left[\begin{array}{lllllll}
0 & 0.57 & 0, & -0.5 & 0.86 & 0, \\
& -0.5 & 0.28 & 0, & -0.33 & 0.57 & 0
\end{array}\right]^{T} } \\
d_{4}= & {\left[\begin{array}{lllllll}
1 & 0 & 0, & 0.5 & 0.28 & 0, \\
& 0.5 & -0.28 & 0, & 0.66 & 0 & 0.47
\end{array}\right]^{T} } \\
d_{5}= & {\left[\begin{array}{lllllll}
-0.33 & 0 & 0.47, & 0.16 & -0.28 & 0.47, \\
0.16 & 0.28 & 0.47, & 0 & 0 & 0
\end{array}\right]^{T} } \\
d_{6}= & {\left[\begin{array}{lllllll}
0.33 & 0 & 3.73, & -0.16 & 0.28 & 3.73, \\
& -0.16 & -0.28 & 3.73, & 0 & 0 & 4.2
\end{array}\right]^{T} }
\end{aligned}
$$

The above formations are identical regular tetrahedrons, and are shown in Fig. 1.

The two-stage time-optimal strategy will now be investigated for both cases of (i) constrained acceleration, and (ii) constrained acceleration and velocity. The values of $a_{\max }$ and $v_{\max }$ are chosen to be $0.1 \frac{\mathrm{m}}{\mathrm{s}^{2}}$ and $0.05 \frac{\mathrm{m}}{\mathrm{s}}$, resulting in $L=0.025 \mathrm{~m}$. In order to find the TOSDF in case (i), the entire convex compact set $\mathscr{C}$ is searched according to Theorem 1, which yields

$$
\begin{aligned}
& \mathscr{S}_{T}=\left[\begin{array}{llllll}
0.3325 & 0 & 0.235, & -0.17 & 0.29 & 0.235,
\end{array}\right. \\
& \left.\begin{array}{lllllll}
-0.17 & -0.29 & 0.235, & 0 & 0 & 0.705
\end{array}\right]^{T}
\end{aligned}
$$

It is also obtained that $T^{(a)}=6.40 \mathrm{~s}$ and $T^{(1 a)}=7.29 \mathrm{~s}$. Thus, the two-stage time-optimal strategy improves the expected value of the reconfiguration time by $12 \%$. The time-optimal special designated formation for this case is depicted in
Fig. 1 by dashed lines. It is noted that the computation time required for finding this formation is 20736 seconds using MATLAB on a 32-bit PC with $2.93 \mathrm{GHz}$ Intel Core Duo CPU.

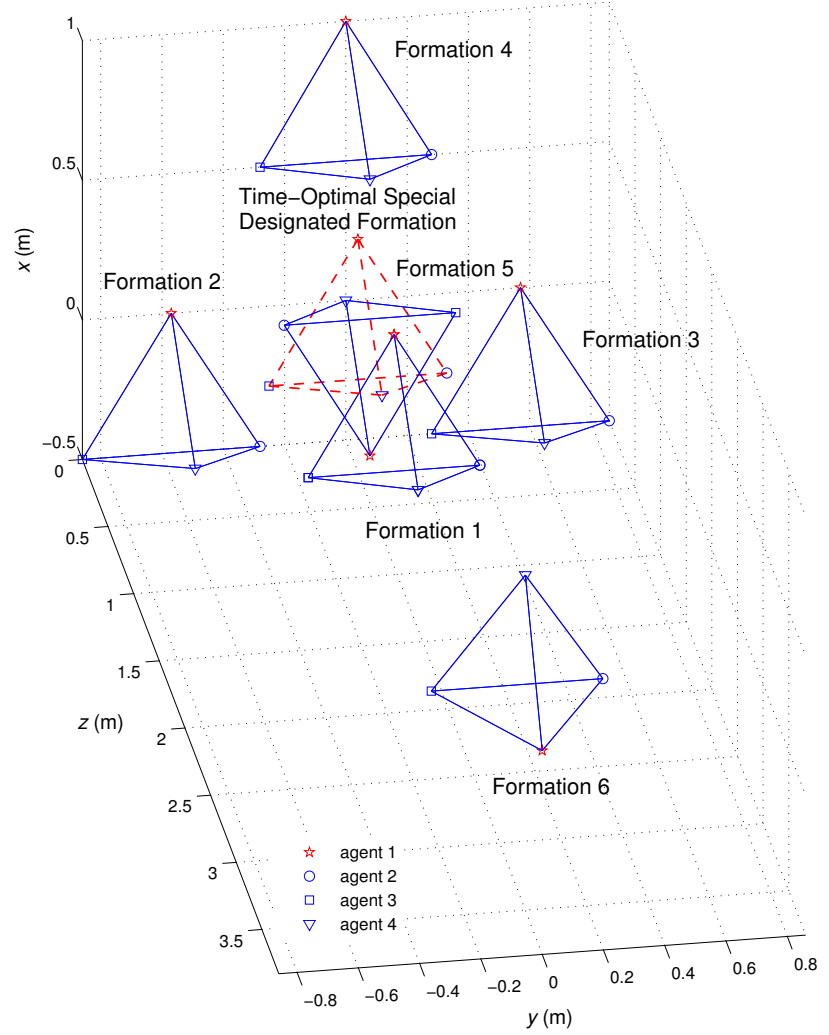

Fig. 1. The six possible formations and the corresponding time-optimal special designated formation.

For case (ii), using the subgradient method the minimum of $J\left(x ; T_{3}\right)$ is obtained to be $J\left(x^{(3)} ; T_{3}\right)=23.89 \mathrm{~s}$. On the other hand, the minimum of $J\left(x ; T_{2}\right)$ on $\mathscr{D}$ is $J\left(x^{\mathscr{D}} ; T_{2}\right)=25.96 \mathrm{~s}$. Since $J\left(x^{\mathscr{D}} ; T_{2}\right)-J\left(x^{(3)} ; T_{3}\right)>2 \frac{v_{\max }}{a_{\max }}=1$, Theorem 5 implies that $x^{(3)}$ given below is a global minimizer of $J\left(x ; T_{2}\right)$.

$$
\begin{aligned}
& x^{(3)}= \\
& {\left[\begin{array}{llllll}
0.3324 & -0.0032 & 0.2434, & -0.1691 & 0.2897 & 0.2413, \\
-0.1686 & -0.2923 & 0.2418, & -0.0034 & -0.0002 & 0.7116
\end{array}\right]^{T}}
\end{aligned}
$$

Moreover, $T^{(v a)}$ is obtained to be $23.89 \mathrm{~s}$, whereas $T^{(1 v a)}$ can be shown to be $34.74 \mathrm{~s}$ for this example. This means that using the two-stage time-optimal strategy $31 \%$ reduction in the expected value of reconfiguration time is achieved. It is to be noted that the TOSDF in this case is obtained without even searching the $L$-vicinity of the formations, using the result of Theorem 5. Since $\mathscr{S}_{T}$ in case (ii) is very close to that of case (i), it is not shown in Fig. 1. The computation 
time required for finding this formation (case (ii)) is only 2 seconds on the same MATLAB platform noted earlier.

\section{Conclusions}

A two-stage time-optimal formation reconfiguration strategy is presented for acceleration-controlled agents. It is assumed that a set of possible formations is given with prescribed probabilities assigned to them, which determines how frequently each formation may occur. In the proposed strategy, the agents are moved to a special designated formation during the idle time in order to minimize the expected value of the reconfiguration time. Finding the special designated formation involves a nonconvex nondifferentiable optimization problem, which is treated for two cases of constrained acceleration, and constrained acceleration and velocity. The maximum achievable reduction in the expected value of the reconfiguration time in both cases is shown to be $50 \%$. A set of local minimizers are derived for both cases, and the search space for finding the special designated formation is reduced to a convex compact set. In the case that both acceleration and velocity are constrained, an alternative search algorithm is presented which is much faster and can even further simplify the problem to a convex nondifferentiable optimization under certain conditions. Simulations demonstrate the efficacy of the proposed method.

\section{Appendix}

Proof of Lemma 1. Define the function $G: \mathbb{R}^{3 n} \rightarrow \mathbb{R}$ as follows

$$
G(x)=\max _{j \in \mathbb{N}_{n}} g\left(x_{j}\right)
$$

It can be shown that the directional derivative of $G$ at $x$ in the direction $u$ is given by

$$
G^{\prime}(x ; u)=\max _{j \in I(x)} g^{\prime}\left(x_{j} ; u_{j}\right)
$$

where $I(x)$ denotes the active indices at $x$, i.e. $I(x)=$ $\left\{j \mid g\left(x_{j}\right)=G(x)\right\}$ (See Danskin's Theorem [5] or Proposition 2.3.2 in [6] for similar results). It follows from (24) that

$$
G^{\prime}(0 ; u)=\max _{j \in I} g^{\prime}\left(0 ; u_{j}\right)
$$

where in this case $I=\mathbb{N}_{n}$, since all $x_{j}$ 's are equal to zero. Therefore, it can be concluded from condition (ii) that $G^{\prime}(0 ; u)=+\infty$ for any nonzero $u$, on noting that a nonzero $u$ implies at least one of the $u_{j}$ 's is nonzero. For $x \neq 0$, on the other hand, $\left\|G^{\prime}(x ; u)\right\|<\infty$; this follows from (24), condition (iii), and the fact that if $x \neq 0$, then $x_{j} \neq 0$ for some $j \in I$ (due to condition (i)).

The above discussion together with the fact that $f^{\prime}(x ; u)=$ $\sum_{d \in \mathscr{D}} p(d) G^{\prime}(x-d ; u)$ implies that for any $x \in \mathscr{D}$, the directional derivative of $f$ in any direction is $+\infty$, and this completes the proof.
Proof of Lemma 2. Consider the function $G$ defined in (23). It is easy to verify that if $g$ satisfies the conditions of the lemma, then $G$ also satisfies these conditions. This implies that $G(x-y) \leq G(x)+G(y)$, for all $x, y \in \mathbb{R}^{3 n}$. Since $p(\tilde{d}) \geq$ 0.5 , one can conclude that $p(\tilde{d}) \geq \sum_{\substack{d \in \mathscr{Q} \\ d \neq \tilde{d}}} p(d)$. Now, for any $x \in \mathbb{R}^{3 n}$

$$
\begin{aligned}
f(x) & =\sum_{d \in \mathscr{D}} p(d) G(x-d)=p(\tilde{d}) G(x-\tilde{d})+\sum_{\substack{d \in \mathscr{Q} \\
d \neq \tilde{d}}} p(d) G(x-d) \\
& \geq \sum_{\substack{d \in \mathscr{D} \\
d \neq \tilde{d}}} p(d) G(x-\tilde{d})+\sum_{\substack{d \in \mathscr{D} \\
d \neq \tilde{d}}} p(d) G(x-d) \\
& \geq \sum_{\substack{d \in \mathscr{d} \\
d \neq \tilde{d}}} p(d) G(\tilde{d}-d)=f(\tilde{d})
\end{aligned}
$$

This completes the proof.

Proof of Lemma 3. To prove this lemma, it suffices to show that for any $x \notin \mathscr{C}$, there is a point $\tilde{x} \in \mathscr{C}$ for which $f(\tilde{x}) \leq$ $f(x)$. Suppose that $x \notin \mathscr{C}$; then $x_{j} \notin \mathscr{C}_{j}$, for some $j \in \mathbb{N}_{n}$. Let $\tilde{x}_{j}$ be the closest point in $\mathscr{C}_{j}$ to $x_{j}$. Using the convex projection theorem (see [9]), it is straightforward to show that for any point $d_{j} \in \mathscr{D}_{j},\left\|x_{j}-d_{j}\right\| \geq\left\|\tilde{x}_{j}-d_{j}\right\|$. Hence, for the point $\tilde{x}$ obtained from $x$ after replacing $x_{j}$ with $\tilde{x}_{j}$, the relation $f(\tilde{x}) \leq f(x)$ holds. On the other hand, the point $\tilde{x}_{j}$ corresponding to $\tilde{x}$ belongs to $\mathscr{C}_{j}$. Therefore, by repeating the same procedure for all those indices $j$ for which $x_{j} \notin \mathscr{C}_{j}$, one can obtain a point $\tilde{x}$ lying in $\mathscr{C}$ such that the value of $f$ at $\tilde{x}$ is not greater than that at $x$.

Proof of Lemma 4. One can easily verify that $G(x+y) \leq$ $G(x)+G(y)$ for all $x, y \in \mathbb{R}^{3 n}$, where $G$ is defined in (23). Thus, for any $x \in \mathbb{R}^{3 n}$ and any $d \in \mathscr{D}, G(x-d) \leq G(x-$ $\left.x^{*}\right)+G\left(x^{*}-d\right)$. Therefore,

$$
\sum_{d \in \mathscr{D}} p(d) G(x-d) \leq \sum_{d \in \mathscr{D}} p(d) G\left(x-x^{*}\right)+\sum_{d \in \mathscr{D}} p(d) G\left(x^{*}-d\right)
$$

which implies that

$$
f(x) \leq G\left(x-x^{*}\right)+f\left(x^{*}\right)
$$

for any $x \in \mathbb{R}^{3 n}$. Using (28), one can write

$$
\sum_{d \in \mathscr{D}} p(d) f(d) \leq \sum_{d \in \mathscr{D}} p(d) G\left(d-x^{*}\right)+\sum_{d \in \mathscr{D}} p(d) f\left(x^{*}\right)
$$

Now, on noting that the right side of the above relation is equal to $2 f\left(x^{*}\right)$, one arrives at

$$
f\left(x^{*}\right) \geq 0.5 \sum_{d \in \mathscr{D}} p(d) f(d)
$$

The right-hand side relation in (2) is a straightforward consequence of the equality $\sum_{d \in \mathscr{D}} p(d)=1$ and the fact that $f(d) \geq f\left(x^{*}\right)$ for all $d \in \mathscr{D}$, as $x^{*}$ is a global minimizer of $f$. 


\section{References}

[1] Amir Ajorlou, Kaveh Moezzi, Amir G. Aghdam, Siamak Tafazoli, and Sergey G. Nersesov. Two-stage energy-optimal formation reconfiguration strategy. Automatica, 2012. http://dx.doi. org/10.1016/j.automatica.2012.06.059.

[2] M. L. Balinski, P. M. Wolfe, and D. P. Bertsekas. Nondifferentiable Optimization. North-Holland Publishing Company, 1975.

[3] R. W. Beard and F. Y. Hadaegh. Fuel optimized rotation for satellite formations in free space. In Proceedings of American Control Conference, pages 2975-2979, 1999.

[4] D. P. Bertsekas. Nonlinear Programming. Athena Scientific, 1999.

[5] D. P. Bertsekas, A. Nedic, and A. E. Ozdaglar. Convex Analysis and Optimization. Athena Scientific, 2003.

[6] Jonathan M. Borwein and Adrian S. Lewis. Convex Analysis and Nonlinear Optimization: Theory and Examples. Birkhuser, 2006.

[7] S. P. Boyd and C. H. Barratt. Linear Controller Design: Limits of Performance. Prentice-Hall, 1991.

[8] D. W. Casbeer, D. B. Kingston, R. W. Beard, and T. W. Mclain. Cooperative forest fire surveillance using a team of small unmanned air vehicles. International Journal of Systems Science, 37(6):351360, 2006

[9] Kenneth R. Davidson and Allan P. Donsig. Real Analysis and Applications: Theory in Practice. Springer, 2010.

[10] E. Frazzoli, Z. H. Mao, J. H. Oh, and E. Feron. Resolution of conflicts involving many aircraft via semidefinite programming. AIAA Journal of Guidance, Control and Dynamics, 24(1):79-86, 2001.

[11] J. Hu, M. Prandini, and S. Sastry. Optimal coordinated maneuvers for three dimensional aircraft conflict resolution. AIAA Journal of Guidance, Control and Dynamics, 25(5):888-900, 2002.

[12] Saeid Jafari, Amir Ajorlou, Amir G. Aghdam, and Siamak Tafazoli. Distributed control of formation flying spacecraft using deterministic communication schedulers. In Proceedings of the 49th IEEE Conference on Decision and Control, pages 3084-3090, 2010.

[13] O. Junge and S. Ober-Blöbaum. Optimal reconfiguration of formation flying satellites. In Proceedings of the 44th IEEE Conference on Decision and Control, pages 66-71, 2005.

[14] K. C. Kiwiel. Methods of Descent for Nondifferentiable Optimization. Springer-Verlag, 1985

[15] J. Lavaei, A. Momeni, and A. G. Aghdam. A model predictive decentralized control scheme with reduced communication requirement for spacecraft formation. IEEE Transactions on Control Systems Technology, 16(2):268-278, 2008.

[16] P. R. Lawson. The terrestrial planet finder. In Proceedings of IEEE Aerospace Conference, pages 2005-2011, 2001.

[17] N. Lechevin, C. A. Rabbath, and P. Sicard. Trajectory tracking of leader-follower formations characterized by constant line-of-sight angles. Automatica, 42(12):2131-2141, 2006.

[18] M. B. Milam, N. Petit, and R. M. Murray. Constrained trajectory generation for micro-satellite formation flying. In Proceedings of AIAA Guidance, Navigation and Control Conference, pages 328-333, 2001.

[19] D. P. Scharf, F. Y. Hadaegh, and S. R. Ploen. A survey of spacecraft formation flying guidance and control (part i): guidance. In Proceedings of American Control Conference, pages 1733-1739, 2003.

[20] D. P. Scharf, F. Y. Hadaegh, and S. R. Ploen. A survey of spacecraft formation flying guidance and control (part ii): control In Proceedings of American Control Conference, pages 2976-2985, 2004.
[21] N. Z. Shor. Minimization Methods for Non-Differentiable Functions. Springer Series in Computational Mathematics, 1985.

[22] C. Sultan, S. Seereeram, R. K. Mehra, and F. Y. Hadaegh Energy optimal reconfiguration for large scale formation flying. In Proceedings of American Control Conference, pages 2986-2991, 2004.

[23] C. Xiang and A. G. Aghdam. A two-stage formation flying strategy to reduce the mission time. In Proceedings of IEEE International Conference on System of Systems Engineering, 2007. 